\title{
FANO-MORI ELEMENTARY CONTRACTIONS WITH REDUCIBLE GENERAL FIBER
}

\author{
Gianluca Occhetta and Davide Panizzolo
}

\begin{abstract}
Let $\varphi: X \rightarrow W$ be an elementary divisorial Fano-Mori contraction from a smooth projective variety, defined by a linear system $\left|m\left(K_{X}+\tau L\right)\right|$, with $L$ a $\varphi$-ample line bundle in $\operatorname{Pic}(X), \tau$ a positive integer and $m \gg 0$.

General fibers of such contractions are known to be irreducible if $\tau \geq \operatorname{dim} X-3$ (and so if $\operatorname{dim} X \leq 4$ ). We prove that, if $\tau \geq \operatorname{dim} X-4$, except possibly for one case, a general non trivial fiber is irreducible.

The special case, which can occur when $\operatorname{dim} X=5$, is effective, as we show by an example in the last section of the paper.
\end{abstract}

\section{Introduction}

Let $X$ be a smooth projective variety of dimension $n$ defined over the field of complex numbers; a contraction $\varphi: X \rightarrow W$ is a proper surjective map with connected fibers onto a normal variety $W$. If the canonical bundle $K_{X}$ is not nef then the negative part of the cone of effective 1-cycles $\mathrm{NE}(X)$ is locally polyhedral, by the Cone Theorem, and to every face in this part of the cone, by the Contraction Theorem, is associated a contraction; such contractions are called Fano-Mori contractions or extremal contractions. A Fano-Mori contraction is called elementary if $\rho(X / W)=1$ or equivalently if it is associated to an extremal ray, i.e. to a face of dimension one in $\mathrm{NE}(X)_{K_{X}<0}$; in this case we define the length of the ray to be the minimum anticanonical degree of contracted curves.

A Fano-Mori contraction $\varphi: X \rightarrow W$ is defined by a linear system $\left|m\left(K_{X}+\tau L\right)\right|$, with $L$ a $\varphi$-ample line bundle, $\tau$ a positive integer and $m \gg 0$; the divisor $K_{X}+\tau L$ is called a supporting divisor of the contraction.

The integer $\tau$ is bounded above by $n+1$ if $\varphi$ is of fiber type, i.e. if $\operatorname{dim} W<\operatorname{dim} X$ and by $n-1$ if $\varphi$ is birational; elementary contractions with values of $\tau$ close to the maximum were studied by general adjunction theory [6].

The situation, which is quite simple for the maximum values of $\tau(\varphi$ is the contraction of a projective space to a point in the fiber type case or the blow-up

Mathematics Subject Classification. 14E30, 14J40.

Key words and phrases. Extremal rays, Fano-Mori contractions, Rational curves

Received January 11, 2005; revised April 22, 2005. 
of a smooth point in the birational case) becomes more and more complicated as values of $\tau$ decrease.

Elementary contractions with $\tau \geq n-2$ were classified by Mori [13] in the case of smooth threefolds and by Fujita [8] and Ionescu [9] in the general case, while more recently Kawamata [10], Andreatta and Wiśniewski [4] dealt with the case $n=4$ and $\tau=1$ and gave a complete classification.

To proceed in the classification, i.e. to study either the case $\tau=n-3$ with $n>4$ or $\tau<n-3$, the first step is to consider the general non trivial (i.e. non 0 -dimensional) fiber of the contraction. Nakamura [14] considered the case of Fano-Mori elementary contractions either of fiber type or divisorial, supported by $K_{X}+(n-3) L$.

A new problem arises in the divisorial case: the exceptional divisor of the contraction is known to be irreducible by [11, Proposition 5.1.6], but it is no longer easy to prove that the general non trivial fiber of the contraction is irreducible. Nevertheless in the case $\tau=n-3$ general non trivial fibers of divisorial contractions are actually irreducible, as proved in [14].

In the subsequent case (i.e. $\tau=n-4$ ) proving the irreducibility of a general fiber becomes an hard problem. Actually a new phenomenon occurs: there exist elementary divisorial contractions with reducible general non trivial fibers. This is the subject of our paper; more precisely, we prove the following:

THEOREM 1.1. Let $X$ be a smooth complex projective variety, let $\varphi_{X}: X \rightarrow W$ be a divisorial elementary Fano-Mori contraction supported by $K_{X}+\tau L$ and denote by $E$ the exceptional divisor. Suppose that $\tau \geq \operatorname{dim} X-4$. Then the general non trivial fiber of $\varphi_{X}$ is irreducible, except in the following effective case:

1. $\operatorname{dim} X=5$;

2. $\tau=1$;

3. the length of the extremal ray contracted by $\varphi_{X}$ is one;

4. the image of $E, \varphi_{X}(E)$ is a curve;

5. a general non trivial fiber is the union of two irreducible components isomorphic to $\mathbf{P}_{\mathbf{P}^{1}}\left(\mathcal{O}^{\oplus 2} \oplus \mathcal{O}(2)\right)$ which meet along a smooth quadric surface.

In section 2 and 3 we recall general definitions and properties of families of rational curves and Fano-Mori contractions; then, in section 4, we explain the vertical slicing costruction which allows us to reduce to the case of a non elementary divisorial contraction $\varphi: Y \rightarrow Z$ whose exceptional locus is a reducible divisor contracted to a point and such that $\operatorname{dim} Y<\operatorname{dim} X$.

In section 5 we study families of rational curves which cover the irreducible components of the exceptional locus of $\varphi$; the key observation is to consider deformation of curves in the irreducible components of the exceptional locus rather than in the ambient variety. As a byproduct we obtain in this section a different proof of the irreducibility of the general fiber in the case studied in [14]. Section 6 contains the proof of theorem 1.1, while in the last section is presented an example, suggested by Jaroslaw Wiśniewski, which shows the effectiveness of the result. 


\section{Families of rational curves}

Throughout this section our main reference is [12], with which our notation is coherent.

Let $X$ be a projective scheme and let $\operatorname{Hom}\left(\mathbf{P}^{1}, X\right)$ be the scheme parametrizing morphisms from $\mathbf{P}^{1}$ to $X$. Let $\operatorname{Hom}_{b i r}\left(\mathbf{P}^{1}, X\right) \subset \operatorname{Hom}\left(\mathbf{P}^{1}, X\right)$ be the open subscheme corresponding to those morphisms which are birational onto their image and let $\operatorname{Hom}_{b i r}^{n}\left(\mathbf{P}^{1}, X\right)$ be the normalization of $\operatorname{Hom}_{b i r}\left(\mathbf{P}^{1}, X\right)$ : the group $\operatorname{Aut}\left(\mathbf{P}^{1}\right)$ acts on $\operatorname{Hom}_{b i r}^{n}\left(\mathbf{P}^{1}, X\right)$ and the quotient exists.

Definition 2.1. We define the space $\operatorname{RatCurves}^{n}(X)$ to be the quotient of $\operatorname{Hom}_{b i r}^{n}\left(\mathbf{P}^{1}, X\right)$ by the action of $\operatorname{Aut}\left(\mathbf{P}^{1}\right)$ and the space $\operatorname{Univ}(X)$ to be the quotient of the product action of $\operatorname{Aut}\left(\mathbf{P}^{1}\right)$ on $\operatorname{Hom}_{b i r}^{n}\left(\mathbf{P}^{1}, X\right) \times \mathbf{P}^{1}$.

We have the following commutative diagram:

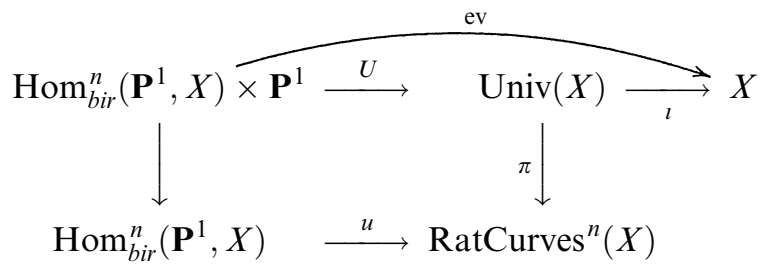

where $u$ and $U$ are principal $\operatorname{Aut}\left(\mathbf{P}^{1}\right)$-bundles, $\pi$ is a $\mathbf{P}^{1}$-bundle and ev is the evaluation map.

There exists a "pointed" version of this construction: let $x \in X$ be a point and let $\operatorname{Hom}_{b i r}\left(\mathbf{P}^{1}, X, 0 \mapsto x\right)$ be the scheme that parametrizes morphisms $f$ : $\mathbf{P}^{1} \rightarrow X$ which send the point $0 \in \mathbf{P}^{1}$ to $x \in X$. Let $\operatorname{Aut}\left(\mathbf{P}^{1}, 0\right)$ be the group of the automorphisms of $\mathbf{P}^{1}$ which fix a point $0 \in \mathbf{P}^{1}$ and let $\operatorname{Hom}_{b i r}^{n}\left(\mathbf{P}^{1}, X, 0 \mapsto x\right)$ be the normalization of $\operatorname{Hom}_{b i r}\left(\mathbf{P}^{1}, X, 0 \mapsto x\right)$ : the group $\operatorname{Aut}\left(\mathbf{P}^{1}, 0\right)$ acts on $\operatorname{Hom}_{b i r}^{n}\left(\mathbf{P}^{1}, X, 0 \mapsto x\right)$ and the quotient exists.

Definition 2.3. The space $\operatorname{RatCurves}^{n}(x, X)$ is the quotient of $\operatorname{Hom}_{b i r}^{n}\left(\mathbf{P}^{1}, X, 0 \rightarrow x\right)$ by the action of $\operatorname{Aut}\left(\mathbf{P}^{1}, 0\right)$ and the space $\operatorname{Univ}(x, X)$ is the quotient of the product action of $\operatorname{Aut}\left(\mathbf{P}^{1}, 0\right)$ on $\operatorname{Hom}_{b i r}^{n}\left(\mathbf{P}^{1}, X, 0 \rightarrow x\right) \times \mathbf{P}^{1}$.

Definition 2.4. A family of rational curves $V$ on $X$ is an irreducible subvariety of $\operatorname{RatCurves}^{n}(X)$. Given a family of rational curves $V$, we can consider the curves of $V$ passing through a fixed point $x \in X$ and call it $V_{x}:=$ $V \cap \operatorname{RatCurves}^{n}(x, X)$.

To each family of rational curves $V$ we can associate its universal family $U$, which is the restriction of $\operatorname{Univ}(X)$. 


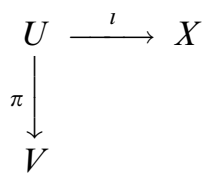

We denote by $\operatorname{Locus}(V)$ the closure of $l(U)$ and we call it the locus of the family; finally we denote by $\operatorname{Locus}\left(V_{x}\right)$ the locus of $V \cap \operatorname{RatCurves}^{n}(x, X)$, i.e. the locus of the curves in the family which pass through $x$.

Definition 2.5. Let $V$ be a family of rational curves on $X$. Then

1. $V$ is unsplit if it is proper;

2. $V$ is locally unsplit if every component of $V_{x}$ is unsplit for a general $x \in \operatorname{Locus}(V)$;

3. $V$ is generically unsplit if there is at most a finite number of curves of $V$ passing through two general points of $\operatorname{Locus}(V)$.

Definition 2.6 [18, Definition 4]. Let $X$ be a projective variety, $H$ an ample divisor on $\mathrm{X}$ and $E \subseteq X$ a closed subset.

A family of rational curves $V$ is a minimal dominating family for $E$ if $E=$ $\operatorname{Locus}(V)$ and $H \cdot V$ is minimal among the families whose locus is $E$.

Remark 2.7. With the above notation, if $V$ is a minimal dominating family for $E$, then $V$ is locally unsplit.

Proposition 2.8 [12, IV.2.6 and II.1.3]. Let $X$ be a projective variety which is a local complete intersection and let $V$ be a family of rational curves whose locus meets the smooth locus of $X$.

Assume either that $V$ is generically unsplit and $x$ is a general point in $\operatorname{Locus}(V)$ or that $V$ is unsplit and $x$ is any point in $\operatorname{Locus}(V)$; then

$$
\operatorname{dim} \operatorname{Locus}(V)+\operatorname{dim} \operatorname{Locus}\left(V_{x}\right)+1 \geq \operatorname{dim} X-K_{X} \cdot V .
$$

What follows is a variation of a classical construction of Mori theory (see for instance [7, Proof of 1.4.5] or [12, II.4.19]).

Definition 2.9. Let $\mathrm{X}$ be a projective variety, let $V$ be an unsplit family of rational curves and let $Y$ be a subset of $X$. We define

$$
\operatorname{Locus}(V)_{Y}:=\{x \in X \mid \exists C \text { in } V \text { with } C \cap Y \neq \emptyset, x \in C\}
$$

i.e. $\operatorname{Locus}(V)_{Y}$ is the set of points that can be joined to $Y$ by a curve of $V$.

Lemma 2.10 [15, Lemma 1]. Let $X$ be a projective variety; let $Y$ be a closed subset of $X$ and let $V$ be an unsplit family of rational curves. Then $\operatorname{Locus}(V)_{Y}$ is closed in $X$ and every curve in Locus $(V)_{Y}$ is numerically equivalent to a linear combination with rational coefficients 


$$
\alpha C_{Y}+\beta C_{V},
$$

where $C_{Y} \subset Y, C_{V}$ belongs to the family $V$ and $\alpha \geq 0$.

COROLlary 2.11. Let $V$ be a family of rational curves on a projective variety $X$, and let $x \in X$ be a point such that an irreducible component of $V_{x}$ is proper and dominant. Then $\rho(X)=1$.

\section{Fano-Mori contractions}

Our notation is coherent with [11], to which we refer for the details.

Let $X$ be a complex projective variety and let

$$
N_{1}(X)=((1-\text { cycles }) / \equiv) \otimes \mathbf{R}, \quad N^{1}(X)=(\operatorname{Pic}(X) / \equiv) \otimes \mathbf{R},
$$

where $\equiv$ denotes numerical equivalence. These vector spaces are dual to each other via the intersection pairing and they are of finite dimension $\rho(X)$, the Picard number of $X$. Let also $\overline{N E(X)} \subset N_{1}(X)$ be the closure of the cone of effective 1-cycles.

From now on we will assume that $X$ is smooth and that $K_{X}$ is not nef, that is there exists an effective curve $C$ such that $K_{X} \cdot C<0$; thus by the Cone Theorem the negative part (with respect to the canonical bundle of $X$ ) of $\overline{N E(X)}$ is locally polyhedral. We will call any face $\sigma$ in the negative part of the cone an extremal face of $X$. By the Rationality Theorem, given an extremal face $\sigma$ of $X$, there exists a nef divisor on $X$ such that $\sigma=\{z \in \overline{N E(X)} \mid H \cdot z=0\} ; H$ is called a supporting divisor for $\sigma$. Moreover, for each extremal face $\sigma$, the Contraction Theorem gives a normal projective variety $W$ and a surjective morphism $\varphi: X \rightarrow W$ with connected fibers such that

1. for every curve $C$ in $X, \varphi(C)$ is a point if and only if the class $[C] \in$ $N_{1}(X)$ is in $\sigma$;

2. $H=\varphi^{*}(A)$, with $A$ an ample Cartier divisor on $W$.

The map $\varphi$ is called Fano-Mori (or extremal) contraction, the contraction of the face $\sigma$, and $H$ is called a supporting divisor for the map $\varphi$. We denote by $\operatorname{Exc}(\varphi)$ the largest subset such that $\varphi$ is an isomorphism on $X \backslash \operatorname{Exc}(\varphi)$ and we call it the exceptional locus of $\varphi$. If the map $\varphi$ is birational, it can be divisorial (if the exceptional locus is a divisor on $X$ ) or small (if the codimension of the exceptional locus in $X$ is greater or equal than 2). If $\operatorname{dim}_{\mathbf{R}} \sigma=1$ the face $\sigma$ is called an extremal ray, while $\varphi$ is called an elementary contraction.

Remark 3.1. We have also (see [13]) that if $X$ has an extremal ray $R$ then there exists a rational curve $\Gamma$ on $X$ such that $0<-K_{X} \cdot \Gamma \leq \operatorname{dim} X+1$ and $R=$ $\mathbf{R}_{+}[\Gamma]$. A rational curve $C$ in $R$ whose intersection number with $-K_{X}$ is minimal is called a minimal extremal curve, while the intersection number $l(R)=-K_{X} \cdot C$ is called length of the ray $R$.

Remark 3.2. We can always choose a supporting divisor of the form $K_{X}+r L$, where $L$ is an ample Cartier divisor and $r$ is an integer. 
Lemma 3.3. Let $\varphi: X \rightarrow W$ be the contraction of an extremal ray $R$; then there exists a locally unsplit family of rational curves $V$ whose numerical class is in $R$, and such that $\operatorname{Locus}(V)=\operatorname{Exc}(\varphi)$.

Proof. $\operatorname{Exc}(\varphi)$ is covered by rational curves whose numerical class is in $R$, so there exists at least an irreducible component of $\operatorname{RatCurves}^{n}(X)$ with these properties; let $V$ be one of these components whose degree with respect to $L$ is minimal. We claim that $V$ is locally unsplit: in fact, if for a general $x \in \operatorname{Exc}(\varphi)$ curves in $V_{x}$ degenerate to a reducible cycle, then the numerical class of every irreducible component of this cycle belongs to $R$ by the extremality of $R$. Then through a general point of $\operatorname{Exc}(\varphi)$ we would have a rational curve of the extremal ray $R$ of degree strictly less than $L \cdot V$, and so a family which covers $\operatorname{Exc}(\varphi)$ with this property, a contradiction.

\section{Divisorial elementary contractions}

In this paper we deal with elementary divisorial contractions, a special case of Fano-Mori contractions, which have the following fundamental property:

Proposition 4.1 [11, Proposition 5.1.6]. Let $\varphi: X \rightarrow W$ be an elementary divisorial Fano-Mori contraction with exceptional divisor $E$; then $E$ is irreducible.

In order to fix more precisely our setup, we give the following

Definition 4.2. Let $X$ be a smooth $n$-fold and let $\varphi_{X}: X \rightarrow W$ be the contraction of an extremal ray $R$, supported by $K_{X}+\tau L$, with $L$ a $\varphi_{X}$-ample line bundle on $X$.

We say that $L$ is numerically reduced on $X$ with respect to $R$ if for every $\varphi_{X^{-}}$ ample line bundle $L^{\prime} \in \operatorname{Pic}(X)$ we have $L \cdot C \leq L^{\prime} \cdot C$ for any curve $C$ whose numerical class is in $R$.

Let $X$ be a smooth $n$-fold and let

$$
\varphi_{X}: X \rightarrow W
$$

be the contraction of an extremal ray $R$. Suppose that $\varphi_{X}$ is divisorial and it is supported by $K_{X}+\tau L$, where $L$ is a $\varphi_{X}$-ample line bundle which is numerically reduced on $X$ with respect to $R$. We denote by $E$ the exceptional divisor, and by $G$ a general non trivial fiber of $\varphi_{X}$, both considered as subschemes of $X$ with the reduced structure.

Lemma 4.3 (See [1, Theorem 2.1] and [14, Lemma 4.2]). The image of the restriction map $\operatorname{Pic}(X) \rightarrow \operatorname{Pic}(G)$ is of rank one, generated by $L_{G}=\left.L\right|_{G}$, and we have

1. $\left.K_{X}\right|_{G}=-\tau L_{G}$;

2. $\left.E\right|_{G}=\left.\left(N_{E / X}\right)\right|_{G}=-q L_{G}$. 
The assumption that $L$ is numerically reduced with respect to $R$ implies that $\tau$ and $q$ are (positive) integers.

To study a general fiber of the contraction $\varphi_{X}: X \rightarrow W$, we will use the vertical slicing technique, following Ando [1]:

4.4 (Construction: Vertical slicing). Let $\varphi_{X}: X \rightarrow W$ be an elementary divisorial Fano-Mori contraction with exceptional divisor $E$ and let $r=\operatorname{dim} \varphi_{X}(E)$. If $r=0$ then the contraction $\varphi_{X}$ maps $E$ to a point and so there is a unique fiber $E$, otherwise we take $r$ general very ample divisors $Z_{1}, \ldots, Z_{r}$ on $W$, we set $Y_{i}=\varphi_{X}^{*}\left(Z_{i}\right)$, and we consider the two varieties

$$
Y:=\bigcap_{i} Y_{i}, \quad Z:=\bigcap_{i} Z_{i}
$$

with $\operatorname{dim} Y=\operatorname{dim} Z=n-r:=m$.

From Bertini theorem we know that $Y$ is smooth and $Z$ is normal; moreover a connected component of $E \cap Y$ is a general fiber $G$ of $\varphi_{X}$. It is straightforward to prove that the restriction of $\varphi_{X}$ to $Y$ is a Fano-Mori contraction $\varphi: Y \rightarrow Z$, supported by $K_{Y}+\tau L_{Y}$, which maps $G$ (a divisor in $Y$ ) to a point in $Z$.

Remark 4.5. If $\varphi_{X}$ contracts $E$ to a point (i.e. if $Y=X$ ), then theorem 1.1 follows immediately from proposition 4.1, so from now on we assume that $m=$ $\operatorname{dim} Y<n=\operatorname{dim} X$.

The normal bundle $N_{G / Y}$ in $Y$ is well defined and we have:

$$
N_{G / Y}=\left.\left(N_{E / X}\right)\right|_{G} \text {. }
$$

Combining this with lemma 4.3, we have

$$
\left.K_{X}\right|_{G}=-\tau L_{G},\left.\quad G\right|_{G}=N_{G / Y}=\left.\left(N_{E / X}\right)\right|_{G}=-q L_{G} .
$$

Suppose that $G$ is reducible:

$$
G=G_{1}+\cdots+G_{s} \text { with } s>1 ;
$$

in this case, by proposition $4.1, \varphi$ can not be elementary, so $\varphi$ is the contraction of an extremal face $\sigma \subset \mathrm{NE}(Y)$ such that $\operatorname{dim} \sigma \geq 2$. Let $R_{1}, \ldots, R_{t}$ be the extremal rays in $\sigma$ and for every $i=1, \ldots, t$ denote by $\varphi_{i}: Y \rightarrow Z_{i}$ the contraction of the ray $R_{i}$. We have commutative diagrams:

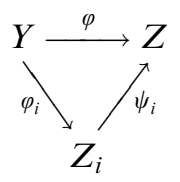

so the contractions $\varphi_{i}$ are birational and supported by $K_{Y}+\tau L_{i}^{\prime}$, with $L_{i}^{\prime}$ a $\varphi_{i}{ }^{-}$ ample divisor on $Y$ which can be written as $L_{i}^{\prime}=L+\varphi_{i}^{*}\left(A_{i}\right)$ for a suitable ample divisor $A_{i} \in \operatorname{Pic}\left(Z_{i}\right)$. 
For every $i=1, \ldots, s$ we can consider the decomposition of $G$

$$
G=G_{i}+\tilde{G}_{i},
$$

where we denote by $\tilde{G}_{i}$ the sum of all the components of $G$ different from $G_{i}$. Since $G$ is connected and reducible, $\tilde{G}_{i}$ is non empty and the restriction

$$
D_{i}:=\left.\tilde{G}_{i}\right|_{G_{i}} .
$$

is an effective divisor on $G_{i}$.

Using the formulas 4.6, we can compute the normal bundle of $G_{i}$ in $Y$ :

$$
N_{G_{i} / Y}=\left.G_{i}\right|_{G_{i}}=\left.\left(G-\tilde{G}_{i}\right)\right|_{G_{i}}=-q L_{G_{i}}-D_{i}
$$

and so, by adjunction, we have

$$
K_{G_{i}}=-(\tau+q) L_{G_{i}}-D_{i}
$$

\section{Rational curves and reducible fibers}

Using the vertical slicing construction we have reduced the study of the general fiber of $\varphi_{X}: X \rightarrow W$ to the study of the exceptional divisor $G$ of a FanoMori contraction $\varphi: Y \rightarrow Z$, from a smooth $m$-fold $Y$, supported by $K_{Y}+\tau L$, whose exceptional locus is a (possibly reducible) divisor $G$ which is mapped to a point. Moreover

$$
K_{G}=-(\tau+q) L \quad N_{G / Y}=-q L
$$

for some positive integers $\tau$ and $q$.

In order to understand the geometry of $G$, in this section we will analyze the properties of the families of rational curves which cover it. The main idea is to use deformations of rational curves in the irreducible components of $G$, as they can give stronger restrictions than deformations in $Y$.

Let $V$ be a generically unsplit family of rational curves in $G_{i}$, such that there exists a curve $C$ in $V$ which meets the smooth locus of $G_{i}$. By proposition 2.8, we have

$$
\operatorname{dim} \operatorname{Locus}(V)+\operatorname{dim} \operatorname{Locus}\left(V_{x}\right)+1 \geq \operatorname{dim} G_{i}-K_{G_{i}} \cdot C,
$$

so, recalling formula 4.8 , we obtain the following inequality:

$$
\operatorname{dim} \operatorname{Locus}(V)+\operatorname{dim} \operatorname{Locus}\left(V_{x}\right)+1 \geq m-1+(\tau+q) L \cdot C+D_{i} \cdot C .
$$

Remark 5.2. Let $V$ be an unsplit family of rational curves in $G_{i}, C$ a curve of the family and $x$ a point of $C$. Since $D_{i}$ is an effective divisor on $G_{i}$, if $D_{i} \cdot C=0$, we have that $\operatorname{Locus}\left(V_{x}\right) \subset D_{i}$.

Proposition 5.3. Let $\varphi: Y \rightarrow Z$ be a Fano-Mori contraction from a smooth $m$-fold $Y$, supported by $K_{Y}+\tau L$, whose exceptional locus is a divisor $G$ which is mapped to a point and such that

$$
K_{G}=-(\tau+q) L \quad N_{G / Y}=-q L .
$$


Suppose moreover that $G$ is reducible and that $\tau \geq m-3$; then

1. each irreducible component $G_{i}$ of $G$ is covered by an unsplit family of rational curves $W^{i}$ such that $L \cdot W^{i}=1$. Moreover the families $W^{j}$ and $W^{k}$ are independent in $N_{1}(Y)$ if $j \neq k$;

2. if $G_{j, k}$ is an irreducible component of $G_{j} \cap G_{k}$, and $x \in G_{j, k}$ is a general point then there exist curves of $W_{x}^{j}$ and $W_{x}^{k}$ which are contained in $G_{j, k}$;

3. every curve in $G_{j, k}$ is numerically equivalent in $Y$ to a linear combination with nonnegative rational coefficients

$$
a \Gamma_{j}+b \Gamma_{k},
$$

where $\Gamma_{j}$ belongs to $W^{j}$ and $\Gamma_{k}$ belongs to $W^{k}$.

Proof of 1. Let $W^{i}$ be a minimal dominating family for $G_{i}$, let $x$ be a general point in $G_{i}$, not contained in Sing $G_{i} \cup D_{i}$ and let $\Gamma_{i}$ be a curve in $W^{i}$ through $x$; since $W^{i}$ is dominating for $G_{i}$ we have $\operatorname{dim} \operatorname{Locus}\left(W^{i}\right)=\operatorname{dim} G_{i}=$ $m-1$, and so inequality (5.1) gives

$$
m \geq \operatorname{dim} \operatorname{Locus}\left(W_{x}^{i}\right)+1 \geq(\tau+q) L \cdot \Gamma_{i}+D_{i} \cdot \Gamma_{i} .
$$

Since $D_{i}$ is effective and $\Gamma_{i} \not \subset D_{i}$ we have $D_{i} \cdot \Gamma_{i} \geq 0$ and so we obtain that

$$
m \geq \operatorname{dim} \operatorname{Locus}\left(W_{x}^{i}\right)+1 \geq(\tau+q) L \cdot \Gamma_{i} \geq(m-2) L \cdot \Gamma_{i} .
$$

Suppose, by contradiction, that $L \cdot \Gamma_{i} \geq 2$; it is easy to show that this can happen only if $m=4, q=1, D_{i} \cdot \Gamma_{i}=0$ and $\operatorname{Locus}\left(W_{x}^{i}\right)=G_{i}$.

Since $W$ is locally unsplit, the last condition, together with corollary 2.11 , gives $\rho\left(G_{i}\right)=1$; in particular $D_{i}$, which is an effective divisor on $G_{i}$, is ample, against the condition $D_{i} \cdot C_{i}=0$. Hence $L \cdot \Gamma_{i}=1$ and $W^{i}$ is an unsplit family.

To prove the independence of $W^{j}$ and $W^{k}$ we note that $G \cdot \Gamma_{i}=-q L \cdot \Gamma_{i}=$ $-q$ and $\operatorname{Locus}\left(W^{i}\right)=G_{i}$ for every $i$; hence $G_{i}$ is negative on $\Gamma_{i}$, and it is the only irreducible component of $G$ with this property. Therefore $G_{j} \cdot \Gamma_{k}<0$ if $j=k$ and $G_{j} \cdot \Gamma_{k} \geq 0$ if $j \neq k$.

Proof of 2. The irreducible components of $G_{j} \cap G_{k}$ are the common components of $D_{j}$ and $D_{k}$, so it is enough to show that for every $i$ and for a general $x$ belonging to an irreducible component $\overline{D_{i}}$ of $D_{i}$ there exists a curve of $W_{x}^{i}$ contained in $\overline{D_{i}}$. We will show that this is the case if $x$ is not contained in any other irreducible component of $D_{i}$.

By inequality 5.5 we have three cases, according to the dimension of $\operatorname{Locus}\left(W_{x}^{i}\right)$.

a) $\operatorname{dim} \operatorname{Locus}\left(W_{x}^{i}\right)=m-3$.

If $\Gamma_{i}$ is a curve in $W_{x}^{i}$, inequality 5.4 gives that $D_{i} \cdot \Gamma_{i}=0$; from remark 5.2 we get $\operatorname{Locus}\left(W_{x}^{i}\right) \subset D_{i}$ and so, by our choice of $x$, $\operatorname{Locus}\left(W_{x}^{i}\right) \subset \overline{D_{i}}$ and we are done.

b) $\operatorname{dim} \operatorname{Locus}\left(W_{x}^{i}\right)=m-2$.

If $\Gamma_{i}$ is a curve in $W_{x}^{i}$, inequality 5.4 gives that either $D_{i} \cdot \Gamma_{i}=0$ or $D_{i} \cdot \Gamma_{i}=1$. 
In the first case, $\operatorname{Locus}\left(W_{x}^{i}\right) \subset D_{i}$ by remark 5.2 and we conclude as in a).

If $D_{i} \cdot \Gamma_{i}=1$, let $G_{l}$ be an irreducible component of $G$, different from $G_{i}$ and containing $\overline{D_{i}}$; since $G_{i} \cap G_{l} \subset D_{i}$ and $\operatorname{Locus}\left(W_{x}^{i}\right) \subset G_{i}$,

$$
\operatorname{Locus}\left(W_{x}^{i}\right) \cap G_{l} \subset \operatorname{Locus}\left(W_{x}^{i}\right) \cap D_{i} .
$$

By inequality 5.4 we have $m \geq 4$, hence $\operatorname{dim} \operatorname{Locus}\left(W_{x}^{i}\right)+\operatorname{dim} G_{l}>\operatorname{dim} Y$, and from Serre's inequality we have that $\operatorname{Locus}\left(W_{x}^{i}\right) \cap D_{i}$ contains a curve; in particular there exists a point $y \neq x \in \operatorname{Locus}\left(W_{x}^{i}\right) \cap D_{i}$, hence a curve $\Gamma$ in $W_{x}^{i}$ which passes through $y$. Being $D_{i} \cdot \Gamma=1, \Gamma$ must be contained in $D_{i}$ (hence in $\overline{D_{i}}$, by our choice of $x$ ) and we are done.

Claim. Case c), i.e. $\operatorname{dim} \operatorname{Locus}\left(W_{x}^{i}\right)=m-1$ cannot happen for any $x$ in $G_{i}$.

Suppose by contradiction that for some $x \in G_{i}$ we have $\operatorname{dim} \operatorname{Locus}\left(W_{x}^{i}\right)=$ $m-1$. This implies $\operatorname{Locus}\left(W_{x}^{i}\right)=G_{i}$ and, since $W_{x}^{i}$ is unsplit, $\rho\left(G_{i}\right)=1$ by corollary 2.11. In particular every curve in $G_{i}$ (and so in $\overline{D_{i}}$ ) is numerically proportional to curves of $W^{i}$.

Let $G_{l}$ be an irreducible component of $G$ containing $\overline{D_{i}}$ and different from $G_{i}$, let $x^{\prime} \in \overline{D_{i}}$ be a generic point and consider Locus $\left(W_{x^{\prime}}^{l}\right)$.

If $\operatorname{dim} \operatorname{Locus}\left(W_{x^{\prime}}^{l}\right)=m-3$ or $m-2$ then $\overline{D_{i}}$ contains curves of $W_{x^{\prime}}^{l}$ by cases a) and b), while if $\operatorname{dim} \operatorname{Locus}\left(W_{x^{\prime}}^{l}\right)=m-1$ every curve in $\overline{D_{i}}$ is numerically proportional to curves of $W_{x^{\prime}}^{l}$ as before.

In all cases this leads to a contradiction with the fact that every curve in $\overline{D_{i}}$ is numerically proportional to curves of $W^{i}$, while $W^{i}$ and $W^{l}$ are numerically independent.

Proof of 3. Let $x$ be a general point of $G_{j, k}$ (i.e. a point which is not contained in any other irreducible component of $\left.G_{j} \cap G_{k}\right)$ and let $\Gamma_{j}$ be a curve of $W_{x}^{j}$ contained in $G_{j, k}$; by our choice of $x$ and $\Gamma_{j}$, the generic point $x^{\prime}$ of $\Gamma_{j}$ is not contained in any other irreducible component of $G_{j} \cap G_{k}$.

Consider Locus $\left(W^{k}\right)_{\Gamma_{j}} \subseteq G_{k}$ (see Definition 2.9): this is a closed subset of $X$ by lemma 2.10 and, by [3, Lemma 5.4] we have

$$
\operatorname{dim} \operatorname{Locus}\left(W^{k}\right)_{\Gamma_{j}}=\operatorname{dim} \operatorname{Locus}\left(W_{x}^{k}\right)+1 .
$$

Therefore according to the proof of part 2 we have two possibilities, depending on the dimension of $\operatorname{Locus}\left(W_{x}^{k}\right)$ :

if $\operatorname{dim} \operatorname{Locus}\left(W_{x}^{k}\right)=m-2$, we have that $\operatorname{dim} \operatorname{Locus}\left(W^{k}\right)_{\Gamma_{j}}=m-1$, and so $\operatorname{Locus}\left(W^{k}\right)_{\Gamma_{j}}^{x}=G_{k}$

if $\operatorname{dim} \operatorname{Locus}\left(W_{x}^{k}\right)=m-3$, we have that $\operatorname{dim} \operatorname{Locus}\left(W^{k}\right)_{\Gamma_{j}}=m-2$ and, if $x^{\prime}$ is

a generic point of $\Gamma_{j}$, Locus $\left(W_{x^{\prime}}^{k}\right)$ is contained in $G_{j, k}$ by proof of part 2 case a). Thus Locus $\left(W^{k}\right)_{\Gamma_{j}} \cap G_{j, k}$ contains a dense subset of $G_{j, k}$ (that is $\bigcup_{x^{\prime}} \operatorname{Locus}\left(W_{x^{\prime}}^{k}\right)$ with $x^{\prime}$ general in $\left.\Gamma_{j}\right)$ and so $G_{j, k} \subset \operatorname{Locus}\left(W^{k}\right)_{\Gamma_{j}}$.

In both cases, we have that $G_{j, k} \subseteq \operatorname{Locus}\left(W^{k}\right)_{\Gamma_{j}}$. 
Let $C$ be a curve in $G_{j, k}$; by Lemma $2.10 C$ is numerically equivalent to a linear combination

$$
C \equiv a_{j} \Gamma_{j}+b_{j} \Gamma_{k},
$$

of curves in $W^{j}$ and $W^{k}$, with $a_{j}, b_{j} \in \mathbf{Q}$ and $a_{j} \geq 0$. We can repeat all the argument exchanging $j$ and $k$ to show that there exist $a_{k}, b_{k} \in \mathbf{Q}$, with $b_{k} \geq 0$, such that

$$
C \equiv a_{k} \Gamma_{j}+b_{k} \Gamma_{k} .
$$

Since $\left[\Gamma_{j}\right],\left[\Gamma_{k}\right] \in N_{1}(Y)$ are linearly independent, the decomposition of $[C]$ is unique, and so

$$
a=a_{j}=a_{k} \geq 0, \quad b=b_{j}=b_{k} \geq 0
$$

as claimed.

COROLlary 5.6. With the assumptions and the notations of proposition 5.3 we have $\tau=m-3$ and $\left.G\right|_{G}=-L_{G}$ i.e. $q=1$.

Proof. By the claim in the proof of proposition 5.3, part 2, for every $x \in G_{i}$ we have $\operatorname{dim} \operatorname{Locus}\left(W_{x}^{i}\right) \leq m-2$; therefore we can rewrite inequality 5.4 as follows:

$$
m-1 \geq \operatorname{dim} \operatorname{Locus}\left(W_{x}^{i}\right)+1 \geq \tau+q+D_{i} \cdot \Gamma_{i} .
$$

Suppose, by contradiction, that $q>1$, or $\tau \geq m-2$; this can happen only if

$$
\operatorname{dim} \operatorname{Locus}\left(W_{x}^{i}\right)=m-2 \text { and } D_{i} \cdot \Gamma_{i}=0 .
$$

In particular, if we choose $x^{\prime} \in D_{i}$, by remark 5.2 we have that $\operatorname{Locus}\left(W_{x^{\prime}}^{i}\right)$ is a closed subscheme of $D_{i}$ with the same dimension and so it is an irreducible component $\overline{D_{i}}$ of $D_{i}$ and has Picard number one by corollary 2.11 .

By proposition 5.3, part 2 we have that each component of $D_{i}$ contains curves of two independent unsplit families and so its Picard number cannot be one, a contradiction.

COROLlary 5.7 [14, Lemma 8.1]. Let $X$ be a smooth complex projective variety of dimension $n$ and let $\varphi_{X}: X \rightarrow W$ be a divisorial elementary Fano-Mori contraction supported by $K_{X}+\tau L$, with $\tau \geq n-3$. Then the general fiber $G$ of $\varphi_{X}$ is irreducible.

Proof. Suppose by contradiction that $G$ is reducible. By vertical slicing we reduce to a Fano-Mori contraction $\varphi: Y \rightarrow Z$ from a smooth $m$-fold $Y$ which satisfies the assumptions of proposition 5.3.

As noted in remark 4.5, we can assume that $m<n$, so, by corollary 5.6 we have $\tau=m-3<n-3$, a contradiction. 


\section{Proof of theorem 1.1}

We are now ready to use the results of the previous sections to prove the main theorem. By vertical slicing we reduce to the study of the following situation:

6.1 (Setup). Let $\varphi: Y \rightarrow Z$ be a Fano-Mori contraction from a smooth $m$-fold $Y$, with $m=\operatorname{dim} Y<\operatorname{dim} X=n$, supported by $K_{Y}+\tau L$, with $\tau \geq n-4$, whose exceptional locus is a reducible divisor $G$ which is mapped to a point and such that $K_{G}=-(\tau+q) L$ and $N_{G / Y}=-q L$.

Since $m<n$ we have that $\tau \geq m-3$, so, by corollary 5.6 we have $\tau=m-3$ and $q=1$, hence

$$
K_{G}=-(m-2) L \quad N_{G / Y}=-L .
$$

\section{STEP 1. Elementary contractions of a vertical section}

As we have seen in section four, $\varphi$ is the contraction of an extremal face $\sigma=\left\langle R_{1}, \ldots, R_{t}\right\rangle$ of dimension $\geq 2$. The contraction $\varphi_{i}: Y \rightarrow Z_{i}$ associated to the ray $R_{i}$ is supported by $K_{Y}+(m-3) L_{i}^{\prime}$ where $L_{i}^{\prime}$ is $\varphi_{i}$-ample and $L_{i}^{\prime}=$ $L+\varphi_{i}^{*} A_{i}$ for a suitable $A_{i}$ ample on $Z_{i}$.

The following lemmata describe some properties of these contractions.

LEMMA 6.2. In the assumptions of Setup 6.1 suppose that one of the contractions $\varphi_{i}$, call it $\varphi_{1}$, is divisorial; then every fiber of $\varphi_{1}$ has dimension $\leq m-2$.

Proof. By proposition 4.1 the exceptional locus $\operatorname{Exc}\left(\varphi_{1}\right)$ of $\varphi_{1}$ is an irreducible divisor, so, being contained in $G$, it coincides with one of its irreducible components, call it $G_{1}$.

Suppose by contradiction that $\operatorname{dim} \varphi_{1}\left(G_{1}\right)=0$; let $G_{2}$ be an irreducible component of $G$ meeting $G_{1}$; by part 2 of proposition 5.3, $G_{1} \cap G_{2}$ contains curves of $W^{2}$, the unsplit family which covers of $G_{2}$ whose existence is guaranteed by part 1 of proposition 5.3 .

Since a general curve of $W^{2}$ is not contained in $\operatorname{Exc}\left(\varphi_{1}\right)$, curves of $W^{2}$ are not contracted by $\varphi_{1}$; in particular the curves of $W^{2}$ contained in $G_{1} \cap G_{2}$ are not contracted by $\varphi_{1}$, a contradiction.

Lemma 6.3. In the assumptions of Setup 6.1 suppose that one of the contractions $\varphi_{i}$, call it $\varphi_{1}$, is a small contraction, and denote by $C_{1}$ a curve in $R_{1}$ of minimal anticanonical degree. Then $G_{i} \cdot C_{1} \leq 0$ for every $i=1, \ldots, s$.

Proof. Since $\varphi_{1}$ is a small contraction supported by $K_{Y}+(m-3) L_{1}^{\prime}$, by [2, Theorem $\mathrm{A}]$, we have that $\varphi_{1}$ contracts a finite number of disjoint $\mathbf{P}^{m-2}$ to points and that $L_{1}^{\prime} \cdot C_{1}=1$.

Fix one of these projective spaces and call it $P$ : of course $P \subset G$ and so there exists a component $G_{1}$ such that $P \subset G_{1}$; we claim that $P$ does not meet any other irreducible component of $G$. 
Suppose by contradiction that $P$ meets $G_{2} \neq G_{1}$; then by Serre's inequality $G_{2} \cap P$ contains a curve $\Gamma$ of $P$. Hence $\Gamma$ is contained in an irreducible component $G_{1,2}$ of $G_{1} \cap G_{2}$.

Thus from part 3 of proposition 5.3 there exist $\alpha, \beta \in \mathbf{Q}$, with $\alpha, \beta \geq 0$, and $(\alpha, \beta) \neq(0,0)$ such that

$$
\Gamma \equiv \alpha \Gamma_{1}+\beta \Gamma_{2},
$$

where $\Gamma_{i}$ is a curve in an unsplit family of rational curves $W^{i}$ which dominates $G_{i}$.

Since $\Gamma$ is contracted by $\varphi_{1}$ it is extremal in $\operatorname{NE}(Y)$, so at least one among $\Gamma_{1}$ and $\Gamma_{2}$ belongs to the extremal ray $R_{1}$ and this is not possible, since $\operatorname{Locus}\left(W^{i}\right)=G_{i}$ is a divisor for every $i$.

Thus we have shown that each irreducible component of the locus of a small ray meets only one component of $G$ (the one in which it is included); then $G_{i} \cdot C_{1}=0$ for $i \neq 1$. Hence $G_{1} \cdot C_{1}=G \cdot C_{1}=-L \cdot C_{1}=-L_{1}^{\prime} \cdot C_{1}=-1$.

\section{STEP 2. Bounding the number of irreducible components}

Proposition 6.4. Let $\varphi: Y \rightarrow Z$ and $G$ be as in Setup 6.1. Then $m=$ $\operatorname{dim} Y=4, \tau=1$ and $G$ has exactly two irreducible components $G_{1}$ and $G_{2}$. Moreover $G_{1}$ and $G_{2}$ are the exceptional loci of two elementary (divisorial) contractions of length one with every non trivial fiber of dimension two.

Proof. Fix one component $G_{1}$ of $G$. Since $G$ is connected we can find a curve $\Gamma \subset G$ which has positive intersection with $G_{1}$ and is not contained in it. The numerical class of $\Gamma$ belongs to the face $\sigma$, so it is a positive linear combination of numerical classes of extremal rational curves. Therefore we can find a minimal extremal curve, say $C_{2}$, which has positive intersection with $G_{1}$.

From lemma 6.3 we have that $R_{2}=\mathbf{R}_{+}\left[C_{2}\right]$ is a divisorial ray; moreover it is clear that $\operatorname{Locus}\left(R_{2}\right)$ meets $G_{1}$. We claim that $\operatorname{Locus}\left(R_{2}\right) \neq G_{1}$.

In fact, since $C_{2}$ is contained in $G$ we have that $G \cdot C_{2}=-L \cdot C_{2}<0$ and so there exists an irreducible component of $G$, call it $G_{2}$ which is negative on $C_{2}$. In particular we have $\operatorname{Locus}\left(R_{2}\right) \subset G_{2}$.

Obviously $G_{1} \neq G_{2}$, since the intersection numbers with $C_{2}$ have opposite sign. Since $R_{2}$ is divisorial we have $\operatorname{Locus}\left(R_{2}\right)=G_{2}$ and so $G_{i} \cdot C_{2} \geq 0$ for every $i \neq 2$; in particular since $G_{1} \cdot C_{2}>0$ we have that

$$
D_{2} \cdot C_{2}=\left(G_{1}+G_{3}+\cdots+G_{s}\right) \cdot C_{2}>0 .
$$

Let $V^{2}$ be a locally unsplit family of rational curves in $R_{2}$ which covers $G_{2}$ (see lemma 3.3) and let $C$ be a curve of $V^{2}$. Denote by $F_{2}$ a general fiber of $\varphi_{2}$ and let $x$ be a general point of $F_{2}$; by inequality 5.1 and lemma 6.2 we have

$$
m-2 \geq \operatorname{dim} F_{2} \geq \operatorname{Locus}\left(V_{x}^{2}\right) \geq(m-2) L \cdot C+D_{2} \cdot C-1
$$

The curve $C$ belongs to $R_{2}$, so $D_{2} \cdot C>0$, forcing $L \cdot C=D_{2} \cdot C=1$, and $[C]=\left[C_{2}\right]$ in $N_{1}(Y)$. Therefore

$$
L \cdot C_{2}=1=D_{2} \cdot C_{2} .
$$


Inequality 6.6 also gives $\operatorname{dim} F_{2}=m-2$ for a general fiber of $\varphi_{2}$; combining this with lemma 6.2 and with upper semicontinuity of the dimension of the fibers we have that $\operatorname{dim} F_{2}=m-2$ for every non trivial fiber of $\varphi_{2}$.

Note that, by the first equality of 6.7 the extremal ray $R_{2}$ has length one, while from the second equality and from the fact that $G_{1} \cdot C_{2}>0$ inequality 6.5 gives $G_{i} \cdot C_{2}=0$ for all $i \neq 1,2$.

Claim. $G_{1}$ is the only component of $G$ which meets $G_{2}$.

Suppose by contradiction that there exists another irreducible component $G_{3}$ of $G$ which meets $G_{2}$; since $G_{3} \cdot C_{2}=0$, if $x$ is a general point of $G_{2} \cap G_{3}$ then Locus $\left(V_{x}^{2}\right) \subset G_{2} \cap G_{3}$ and, being of the same dimension, it is an irreducible component $G_{2,3}$ of $G_{2} \cap G_{3}$. This forces $\rho\left(G_{2,3}\right)=1$, against part 2 of proposition 5.3, and the claim follows.

Now we fix $G_{2}$ and we repeat the first argument of the proof in step 2 to find a divisorial ray $R_{1}$ whose locus is an irreducible component of $G$ different from $G_{2}$ which meets $G_{2}$; from the claim we have $\operatorname{Locus}\left(R_{1}\right)=G_{1}$.

We can repeat now for $R_{1}$ all the steps of the proof; in the end we find that $G_{2}$ is the only component of $G$ which meets $G_{1}$, so, since $G$ is connected we have that $G$ has exactly two irreducible components.

We have also proved that each of this components is the exceptional locus of an elementary divisorial contraction of length one. To prove that $m=\operatorname{dim} Y=4$ (and so that $\tau=1$ ) we consider a point $x$ in $G_{1} \cap G_{2}$; denoted by $F_{1}$ and $F_{2}$ the fibers of $\varphi_{1}$ and $\varphi_{2}$ containing $x$, by Serre's inequality we have

$$
\operatorname{dim}\left(F_{1} \cap F_{2}\right) \geq \operatorname{dim} F_{1}+\operatorname{dim} F_{2}-\operatorname{dim} Y \geq m-4 .
$$

Thus if $m \geq 5$ there exists a curve whose numerical class belongs to two different extremal rays, and this is impossible.

\section{STEP 3. Description of the irreducible components}

Proposition 6.8. Let $\varphi: Y \rightarrow Z$ and $G$ be as in Setup 6.1; then the two irreducible components $G_{1}$ and $G_{2}$ of $G$ are $\mathbf{P}^{2}$-bundles isomorphic to $\mathbf{P}_{\mathbf{P}^{1}}\left(\mathcal{O}^{\oplus 2} \oplus \mathcal{O}(2)\right)$ which meet along a smooth quadric.

Proof. Denote by $\varphi_{i}$ the divisorial contraction whose locus is $G_{i}$ and by $C_{i}$ a curve in $R_{i}$ which has minimal anticanonical degree; by [5, Theorem 4.1], or [17, Main Theorem], we have two possibilities: $\varphi_{i}$ is either a $\mathbf{P}^{2}$-bundle or a quadric bundle over a smooth curve.

The second case is ruled out because, applying inequality 6.6 to a general point $x$ in a general fiber of $\varphi_{i}$, we have $\operatorname{dim} \operatorname{Locus}\left(V_{x}^{i}\right)=2$. Thus $\varphi_{i}$ gives to $G_{i}$ a $\mathbf{P}^{2}$-bundle structure over a smooth curve $B_{i}$.

Let $G_{1,2}$ be an irreducible component of $G_{1} \cap G_{2}$ (and so of $D_{i}$ ); since $G_{1,2}$ is an effective divisor on $G_{i}$ we have that $G_{1,2} \cdot C_{i} \geq 0$ in $G_{i}(i=1,2)$, equality 
holding if and only if $G_{1,2}$ is a fiber of $\varphi_{i}$, but this possibility is ruled out by part 2 of proposition 5.3 .

Therefore $G_{1,2} \cdot C_{i}>0$; since, by formula 6.7 , we have $D_{i} \cdot C_{i}=1$ we conclude that $D:=D_{1}=D_{2}=G_{1,2}$ is irreducible.

Since $D_{1} \cdot C_{1}=D_{2} \cdot C_{2}=1$ and $D$ is irreducible we see that $D$ restricts to a line on every fiber of $\varphi_{i}$ and so $D$, being a surface with two $\mathbf{P}^{1}$-bundle structures, is isomorphich to $\mathbf{P}^{1} \times \mathbf{P}^{1}$.

Moreover $\mathscr{E}_{i}=\varphi_{i *} D$ is a locally free sheaf of rank three on $B_{i}$, which is rational and $G_{i}=\mathbf{P}_{B_{i}}\left(\mathscr{E}_{i}\right)$. In $G_{i}$ we have

$$
\left(D \cdot C_{j}\right)_{G_{i}}=\left.G_{j}\right|_{G_{i}} \cdot C_{j}=\left(G-G_{i}\right) \cdot C_{j}=-2,
$$

so, pushing forward the exact sequence

$$
0 \rightarrow \mathcal{O}_{G_{i}} \rightarrow \mathcal{O}_{G_{i}}(D) \rightarrow \mathcal{O}_{D}(D) \rightarrow 0
$$

to $B_{i} \simeq \mathbf{P}^{1}$ we obtain the exact sequence

$$
0 \rightarrow \mathcal{O}_{\mathbf{P}^{1}} \rightarrow \mathscr{E}_{i} \rightarrow \mathcal{O}(-2) \oplus \mathcal{O}(-2) \rightarrow 0 .
$$

This sequence splits, hence we have $G_{i}=\mathbf{P}\left(\mathcal{O}_{\mathbf{P}^{1}} \oplus^{2} \mathcal{O}_{\mathbf{P}^{1}}(-2)\right)$.

\section{Conclusion of proof of theorem 1.1}

We have only to show that $\operatorname{dim} X=5$, since this will imply also that $\operatorname{dim} \varphi_{X}(E)=1$, but the assertion is clear, since we have proved that $\tau=1$ and we are assuming $\tau \geq \operatorname{dim} X-4$ and $\operatorname{dim} X>\operatorname{dim} Y=4$.

\section{Example}

We will now present an example, due to Jaroslaw A. Wiśniewski, of an elementary Fano-Mori contraction of a smooth fivefold contracting a divisor to a curve and such that any positive dimensional fiber is reducible (and its structure is as described in theorem 1.1). The construction is divided in two steps: first, using toric geometry, we construct a Fano-Mori divisorial contraction of a fourfold with reducible exceptional locus (corresponding to the situation described in proposition 6.8) and then we fit it in a suitable five dimensional manifold.

\subsection{A Fano Mori contraction with a $\mathbf{Z}_{2}$-action}

Let $e_{1}, e_{2}, v_{1}, v_{2}$ be a basis of a 4-dimensional lattice, and let $w_{1}=2 e_{1}-$ $e_{2}-v_{1}$ and $w_{2}=2 e_{2}-e_{1}-v_{2}$. Let $\Delta$ be the fan generated by these six vectors and containing the following maximal cones:

$$
\begin{array}{llll}
\left\langle e_{1}, v_{1}, w_{1}, v_{2}\right\rangle & \left\langle e_{1}, v_{1}, w_{1}, w_{2}\right\rangle & \left\langle e_{1}, e_{2}, v_{1}, w_{2}\right\rangle & \left\langle e_{1}, e_{2}, v_{1}, v_{2}\right\rangle \\
\left\langle e_{2}, v_{2}, w_{2}, v_{1}\right\rangle & \left\langle e_{2}, v_{2}, w_{2}, w_{1}\right\rangle & \left\langle e_{1}, e_{2}, v_{2}, w_{1}\right\rangle & \left\langle e_{1}, e_{2}, w_{1}, w_{2}\right\rangle
\end{array}
$$

Let $Y$ be the variety associated to this fan and let $Z$ be the affine toric variety associated to the cone $\Sigma=\left\langle v_{1}, v_{2}, w_{1}, w_{2}\right\rangle$. The fan $\Delta$ is a subdivision of $\Sigma$, 
obtained introducing the vectors $e_{1}$ and $e_{2}$; let $\varphi: Y \rightarrow Z$ be the proper birational morphism associated to this subdivision (see figure 1 ).

It is straightforward to prove, using basic toric geometry, that
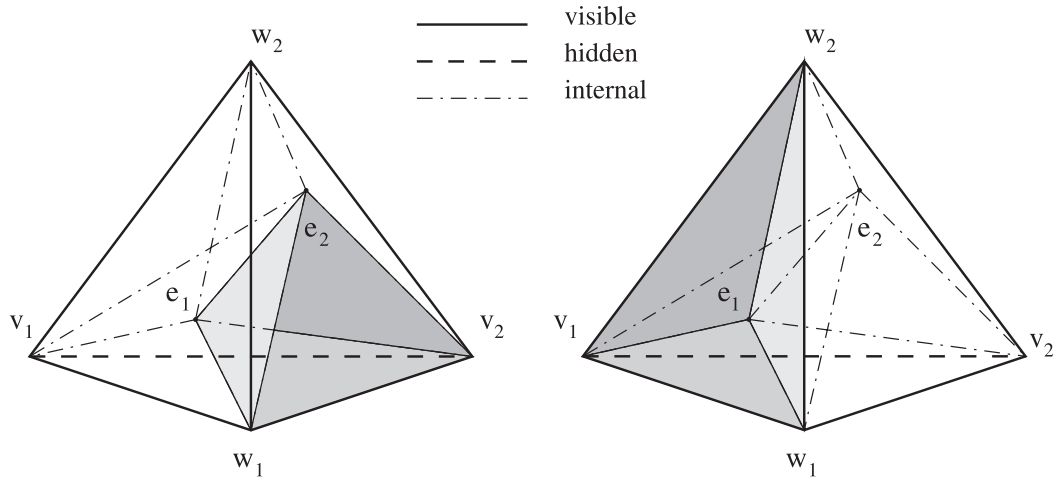

FIgURE 1. The fan $\Delta$ and two elements of the subdivision of $\Delta$

1. $Y$ is smooth;

2. $\operatorname{Exc}(\varphi)$ consists of two divisors $G_{1}$ and $G_{2}$, isomorphic to $\mathbf{P}_{\mathbf{P}^{1}}\left(\mathcal{O}^{\oplus 2} \oplus \mathcal{O}(2)\right)$, which intersect along a quadric $\mathbf{P}^{1} \times \mathbf{P}^{1}$ and are mapped to a point by $\varphi$;

3. $\varphi$ is a Fano-Mori contraction;

4. there is a $\mathbf{Z}_{2}$-action which interchanges $e_{1}$ and $e_{2}, v_{1}$ and $v_{2}, w_{1}$ and $w_{2}$. $N_{1}(G)$ is generated by two classes of rational curves $\left[C_{1}\right]$ and $\left[C_{2}\right]$ : in $G_{1},\left[C_{1}\right]$ is the class of lines in the fibers and $\left[C_{2}\right]$ is the class of a minimal section corresponding to a surjection $\mathcal{O}^{\oplus 2} \oplus \mathcal{O}(2) \rightarrow \mathcal{O}$, while in $G_{2}$ these classes are exchanged; moreover the $\mathbf{Z}_{2}$-action exchanges $G_{1}$ with $G_{2}$ and $\left[C_{1}\right]$ with $\left[C_{2}\right]$.

\subsection{Moebius strip construction}

Let $C^{\prime}$ be a smooth curve with a free $\mathbf{Z}_{2}$-action, so that the action induces an étale covering $C^{\prime} \rightarrow C$ of degree 2. Take the product actions $\pi: Y \times C^{\prime} \rightarrow X$ and $\pi^{\prime}: Z \times C^{\prime} \rightarrow W$, where $X$ and $W$ are the quotients; these product actions are free and so $X$ is smooth.

By the universal property of group actions there exists a morphism $\varphi_{X}: X \rightarrow W$ such that the following diagram commutes:

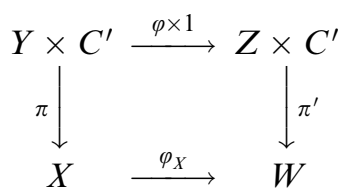

Claim. The map $\varphi_{X}: X \rightarrow W$ is an elementary Fano-Mori contraction, whose exceptional locus is $E=\pi\left(G \times C^{\prime}\right)$ (where $G$ is the exceptional locus of $\varphi$ and $\varphi(G)=z \in Z$ ), which is mapped to $C \subset W$; moreover, a vertical slicing of $\varphi_{X}$ is the contraction $\varphi: Y \rightarrow Z$ constructed in the previous subsection. 

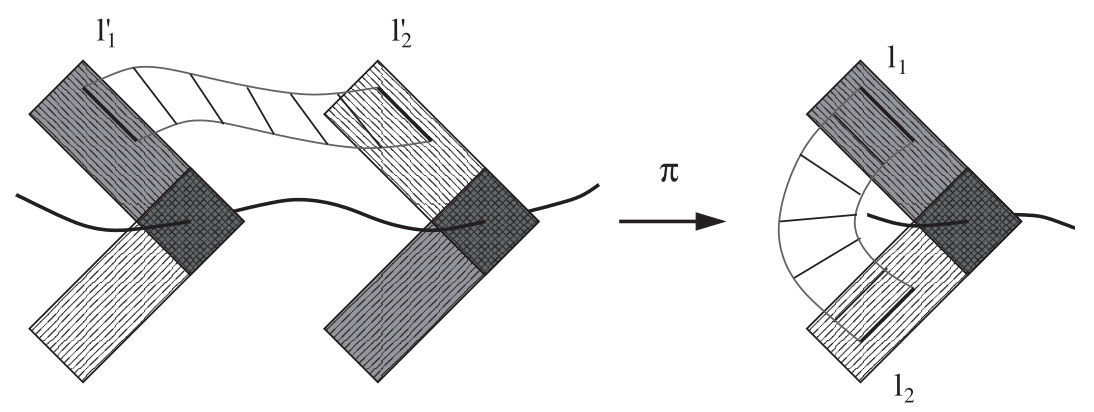

FIGURE 2. $\ell_{1}$ and $\ell_{2}$ are algebraically equivalent

We prove only the non trivial fact that $\varphi_{X}$ is elementary.

Fix $c \in C$, and let $F=\varphi_{X}^{-1}(c)$ be the fiber of $\varphi_{X}$ over $c ; F$ is isomorphic to $G$ via $\pi$ and we denote by $F_{1}$ and $F_{2}$ its irreducible components, which are rational $\mathbf{P}^{2}$-bundles.

We will show that a line in a fiber of $F_{1}$ is algebraically equivalent to a line in a fiber of $F_{2}$; since $N_{1}(F)$ is generated by the classes of such lines, $\varphi_{X}$ is elementary.

Let $\ell$ be a line in a fiber of $G_{1}$ and consider the product $\ell \times C^{\prime} \subset G \times C^{\prime}$ : it is a flat family of rational curves and it is mapped by $\pi$ into the exceptional locus of $\varphi_{X}$. Let $z \times\left\{c_{1}^{\prime}, c_{2}^{\prime}\right\}=\pi^{\prime-1}(c)$, let $\ell_{i}^{\prime}=\ell \times\left\{c_{i}^{\prime}\right\} \subset G_{1} \times\left\{c_{i}^{\prime}\right\}$ and consider the restriction of the previous diagram

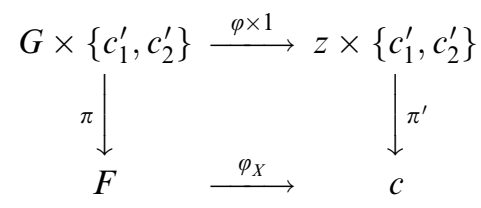

Since the product action identifies $G_{1} \times\left\{c_{1}^{\prime}\right\}$ with $G_{2} \times\left\{c_{2}^{\prime}\right\}$ and $G_{2} \times\left\{c_{1}^{\prime}\right\}$ with $G_{1} \times\left\{c_{2}^{\prime}\right\}$ we have that $\ell_{1}=\pi\left(\ell_{1}^{\prime}\right)$ is a line in a fiber of $F_{1}$ and $\ell_{2}=\pi\left(\ell_{2}^{\prime}\right)$ is a line in a fiber of $F_{2}$.

Acknowledgements. This work grew out of a part of the second named author's PhD thesis [16]; he would like to thank Marco Andreatta who proposed him the problem and supported him during the whole $\mathrm{PhD}$ program; we are also indebted to Edoardo Ballico and Massimiliano Mella for helpful discussions.

The example in section 7, together with some valuable comments and suggestions, was provided by Jaroslaw Wiśniewski within the program of cooperation of Italian and Polish nodes of EAGER European Algebraic Research Training Network, EC grant HPRN-CT-2000-00099. We also would like to thank the anonymous referee whose suggestions made the exposition much clearer. 


\title{
REFERENCES
}

[ 1 ] T. ANDo, On extremal rays of the higher dimensional varieties, Invent. Math. 81 (1985), 347357.

[ 2 ] M. Andreatta, E. Ballico and J. A. Wiśniewski, Two theorems on elementary contractions, Math. Ann. 297 (1993), 191-198.

[ 3 ] M. Andreatta, E. Chierici And G. Occhetta, Generalized Mukai conjecture for special Fano varieties, Cent. Eur. J. Math. 2 (2004), 272-293.

[4] M. Andreatta AND J. A. WiŚnieWski, On contractions of smooth varieties, J. Algebraic Geom. 7 (1998), 253-312.

[ 5 ] M. Andreatta AND J. A. WiŚniewski, Contractions of smooth varieties II: computations and applications, Boll. Unione Mat. Ital., Sez. B. Artic. Ric. Mat. (8) 1 (1998), 343-360.

[6] M. C. Beltrametti AND A. J. Sommese, The adjunction theory of complex projective varieties, Exp. Math. 16, de Gruyter, Berlin, 1995.

[ 7 ] M. C. Beltrametti, A. J. Sommese AND J. A. WiŚniewski, Results on varieties with many lines and their application to adjunction theory, Complex Algebraic Varieties, Bayreuth 1990, Lecture Notes in Math. 1507, Springer-Verlag, 1992, 16-38.

[8] T. FujITA, On polarized manifolds whose adjoint bundles are not semipositive, Algebraic geometry, Sendai, Adv. Studies in Pure Math. 10, Kinokuniya-North-Holland, 1987, 167-178.

[9] P. Ionescu, Generalized adjunction and applications, Math. Proc. Camb. Phil. Soc. 99 (1986), 457-472.

[10] Y. Kawamata, Small contractions of four dimensional algebraic manifold, Math. Ann. 284 (1989), 595-600.

[11] Y. Kawamata, K. Matsuda AND K. Matsuki, Introduction to the minimal model program, Algebraic geometry, Sendai, Adv. Studies in Pure Math. 10, Kinokuniya-North-Holland, 1987, 283-360.

[12] J. Kollár, Rational curves on algebraic varieties, Ergebnisse der Math. 32, Springer Verlag, Berlin, Heidelberg, New York, Tokio, 1996.

[13] S. Mori, Threefolds whose canonical bundles are not numerically effective, Ann. Math. 116 (1982), 133-176.

[14] S. Nakamura, On the third adjoint contractions, J. Reine Angew. Math. 467 (1995), 51-65.

[15] G. OcchetTA, A characterization of products of projective spaces, to appear on Canad. Math. Bull.

[16] D. Panizzolo, Maps between projective varieties, Description of the generic fiber of a FanoMori contraction, UTM PhDTS 35, University of Trento, February 2003.

[17] H. TAKagi, Classification of extremal contractions from smooth fourfolds of $(3,1)$-type, Proc. Amer. Math. Soc. 127 (1999), 315-321.

[18] J. Wierzba AND J. A. Wiśniewski, Small contractions of symplectic 4-folds, Duke Math. J. 120 (2003), 65-95.

\author{
Dipartimento di Matematica \\ Università DEgLi StUdi di Trento \\ VIA SOMMARIVE 14 \\ I-38100 Povo (TN) \\ ITALY \\ E-mail: occhetta@science.unitn.it \\ panizza@science.unitn.it
}

